\title{
Interference Avoidance with Dynamic Inter-Cell Coordination for Downlink LTE System
}

\author{
Mahmudur Rahman ${ }^{\#}$, Halim Yanikomeroglü, and William Wong ${ }^{*}$ \\ ${ }^{\#}$ Department of Systems and Computer Engineering \\ Carleton University, Ottawa, Canada \\ \{mmrahman, halim\}@sce.carleton.ca \\ *Communications Research Centre of Canada (CRC)
Ottawa, Canada
william.wong@crc.ca
}

processing to estimate interference and subtract it from the desired signal component. Interference averaging technique such as frequency hoping ( $\mathrm{FH}$ ) ensures user equipments (UEs) to access a range of channels rather than a narrow set in a specific pattern so that interference effect is averaged out for all UEs. Finally, the interference avoidance technique focuses on finding an optimal effective reuse factor often achieved through restrictions on frequency and power allocations to achieve network performance goals. The benefits of each of these schemes are mutually exclusive; therefore, a combination of the above strategies is expected in future systems. The focus of this paper is on interference avoidance through dynamic inter-cell coordination.

UEs at the cell ${ }^{1}$ border experience high interference from neighbouring transmitters in addition to high path-losses. Scheduling schemes with objective to achieving maximized network throughput find these UEs with poor channel conditions less attractive as they do not contribute much to the total throughput. A blunt approach to improve cell-edge UEs' rates by assigning more resources (i.e., prioritizing cell-edge UEs in a certain way) would jeopardize the rates for other UEs in the cell-centre, and hence, such an approach is highly undesirable. The objective of interference avoidance is to provide better services to cell-edge UEs without sacrificing cell-centre throughput. In this paper, an interference avoidance scheme that uses dynamic inter-cell coordination through X2 interface is investigated.

Inter-cell interference can be reduced significantly with the traditional frequency reuse strategy as presented in the classical article [5] on cellular clustering. Fig. 1 shows reuse of 3 along with reuse of 1 and other static partition-based schemes. The higher the cluster size the greater the reduction in inter-cell interference. However, this improvement in interference can only be realized with the reduction in cell throughput. Although such a traditional reuse scheme might have been good enough to support traffic demands of the early networks, the rate requirements in the future systems warrant more aggressive reuse. In recent years, a large number of available studies in the literature consider reuse partitioning in which cell-edge UEs are assigned resources with higher reuse factors compared to the UEs at the cell-centre to obtain an effective reuse which is somewhat greater than 1 but not too high. In general these schemes are referred to as fractional

\footnotetext{
${ }^{1}$ The terms cell and sector are used interchangeably in this paper; inter-cell interference implies the interference power received from any sector antenna to a UE in a sector of interest.
} 
frequency reuse (FFR) schemes. Evolved from the idea of classical reuse clustering, FFR was first introduced in [6] for Global System for Mobile (GSM) systems. Variants of FFR such as soft frequency reuse (SFR) and partial frequency reuse (PFR) have been adopted in the WiMAX and 3GPP LTE systems (see [7] and [8] for example). This idea is also explored extensively in the WINNER project [9]. While this approach improves interference to the cell edge UEs, there is a great potential to lose overall cell throughput due to resource loss resulted from partitioning.

An optimal partitioning depends on the distribution of the UEs, arrived traffic, and channel dynamism. Therefore, any static reuse partitioning scheme would be a highly sub-optimal solution. A dynamic partitioning based on UEs' traffic load as well as mutual interference situation may provide balanced improvements for both the cell-edge and cell-centre UEs' rates. Such a dynamic reuse adaptation requires dynamic inter-cell coordination. An interference avoidance scheme with dynamic inter-cell coordination is presented for the WINNER system in [10]. The scheme requires a central entity such as radio network controller (RNC). Centralized processing of RRM algorithms is not encouraged in LTE due to the absence of RNC. However, inter-cell coordination among neighbouring cells facilitated through X2 interface [11] is supported in LTE. We modify the scheme presented in [10] to suit to LTE downlink as well as further improve by separating handling of interference originated from the sectors of own eNB and that from the other eNBs. In particular, we present a novel approach to utilize Hungarian algorithm by devising utility matrix in a multi-cell fashion to handle intercell intra-eNB interferers.

The remainder of this paper is organized as follows. Section II gives overview of the available static schemes in the literature. The proposed scheme is described in Section III. Section IV provides LTE simulation environment and parameters. Simulation results are discussed in V followed by conclusions in VI.

\section{STATIC INTERFERENCE AVOIDANCE SCHEMES}

\section{A. Soft frequency reuse}

Soft frequency reuse scheme is a variation of FFR, where the reuse factor is 1 and equal or greater than 1 in the cellcentre and cell-edge areas, respectively. It was proposed in [12] and [13] under 3GPP LTE framework to provide a higher rate to disadvantaged UEs such as those near the cell boundary.

Fig. 1.c shows an example SFR scheme for cell sites with sectorization. For 3 -sector cell sites, the cell edge band is usually $1 / 3$ of the available spectrum and is orthogonal to neighbouring cells. The cell-edge subcarriers are called major subcarrier group while the cell-centre frequency band is termed as minor subcarrier group. The total transmit power is set fixed and each group is assigned transmission power depending on desired effective reuse factor, which is determined by the power ratio of cell-centre to cell-edge groups.
Transmissions use higher power on the major band as shown in the right side of Fig. 1.c. Let us consider that the power per physical resource block (PRB) is 1 in the case of reuse of 1 and power per PRB for the cell-edge (major) band is $\alpha$ for the SFR scheme. Then power per PRB in the minor band would be $(3-\alpha) / 2$ giving a power ratio of $(3-\alpha) / 2 \alpha$. Minor band is available to cell-centre UEs only and major band can also be used for cell centre areas. Adjusting power ratio from 0 to 1 effectively moves the reuse factor from 3 to 1 . Therefore for a tri-sector cell, SFR is a compromise between reuse 1 and 3. UEs are categorized into cell-edge and cellcentre based on user geometry.

\section{B. Partial frequency reuse}

The idea of the partial frequency reuse (PFR) was first presented in [14]. While somewhat similar to SFR in terms of frequency planning, the effective reuse factor of this scheme is always greater than 1 . PFR and its variants are studied in the 3GPP and WINNER projects (see, for example, [9] and [15]). Fig. 1.d shows an example of PFR for sites with sectorization. Let us consider that the total bandwidth is $\beta$ and it is divided to inner zone and outer zone with $\beta_{1}$ and $\beta_{2}$, respectively. For a reuse factor of 3 in the outer zone, the frequency band assigned to each sector's outer zone is $\beta_{2} / 3$. Therefore, the effective frequency reuse is given by $\beta /\left(\beta_{1}+\beta_{2} / 3\right)$. Like SFR, the power used for the outer zone PRBs can be amplified as shown in the figure.

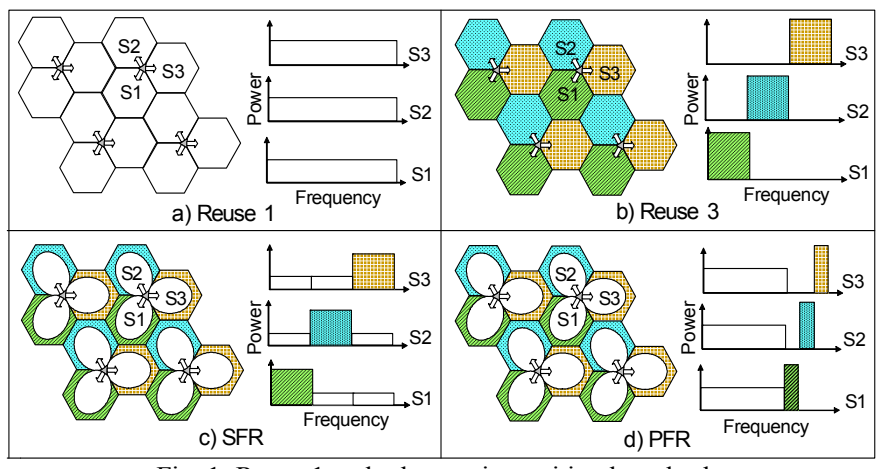

Fig. 1: Reuse 1 and other static partition based schemes

\section{DESCRIPTION OF THE PROPOSED SCHEME}

Inter-cell interference is categorized into two groups, i.e., intra-eNB and inter-eNB interference as shown in Fig. 2. UEs receive dominant interference from the first-tier of interferers, 2 from own-eNB and 4 from cells of other neighbouring eNBs, as illustrated in the figure. Based on mutual interference situation and UE rate requirements resource restrictions are prepared using two algorithms, one for intra-eNB and the other for inter-eNB interferers. Interference originated from cells of own eNB should be handled separately as eNB can take appropriate measures itself without the need for intereNB communication through X2 interface. Both algorithms involve preparation of utility matrix and applying Hungarian algorithm [16] on the utility matrix in an iterative manner in order to find PRBs to be restricted in the neighbouring cells. Hungarian algorithm is optimal for one-to-one PRB to UE 
allocation; however, it becomes sub-optimal when it is used iteratively to assign more than one PRB to a UE.

The restrictions on the usage of PRBs are determined from time-to-time at a time-interval within the channel coherence time, i.e. depending on the speed of the mobile. This interval is denoted as resource restriction refresh interval. Once the $\mathrm{PRB}$ restriction list is available at a sector, the scheduler can perform PRB scheduling based on its own criteria. We studied two variations of the proposed scheme; restricted PRBs are not used at all in one and these PRBs are used, however, only with reduced power (for example, with $10 \mathrm{~dB}$ lower) in another variation.

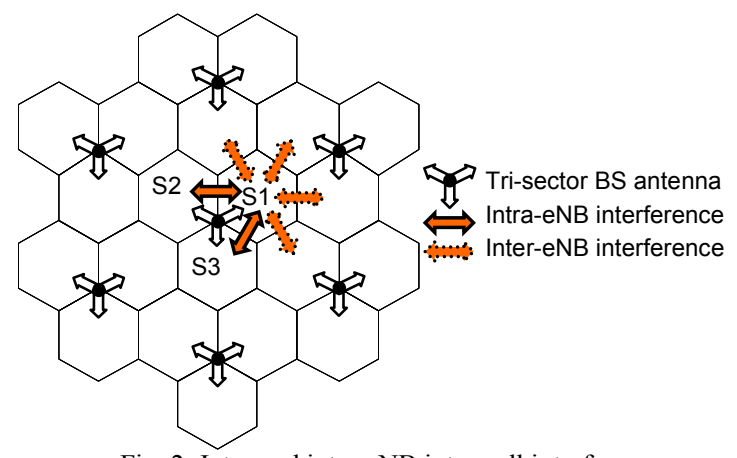

Fig. 2: Intra and inter-eNB inter-cell interference

\section{A. Intra-eNB inter-cell interference avoidance}

For a particular PRB, a sector can restrict only one dominant intra-eNB interferer. A utility matrix covering all three sectors of an eNB is constructed as follows.

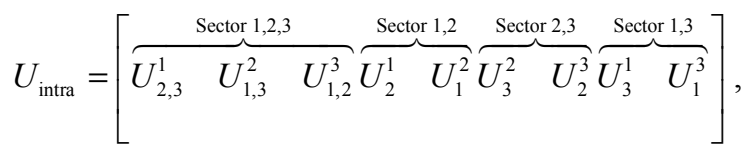

where each element of the above composite matrix is itself a matrix of size $N \times M ; N$ and $M$ are the number of PRBs and the number of UEs per sector, respectively. The first three elements are the utility matrices for sectors 1,2 , and 3 respectively, given remaining sectors (i.e., $\{2,3\},\{1,3\}$, and $\{1,2\})$ use PRBs concurrently. Subsequent subsets of matrices show utility, when one of the intra-eNB sectors is restricted to use PRBs. These utility matrices conditioned on the possible concurrent intra-eNB inter-cell interferers are given by:

$$
U_{i 2, i 3}^{i 1}=\left(\begin{array}{cccc}
u_{1,1} & u_{1,2} & \cdots & u_{1, M} \\
u_{2,1} & u_{2,2} & \cdots & u_{2, M} \\
u_{3,1} & u_{3,2} & \cdots & u_{3, M} \\
\vdots & \vdots & \vdots & \vdots \\
u_{N, 1} & u_{N, 2} & \cdots & u_{N, M}
\end{array}\right) .
$$

The utility measure $u_{n, m}$ is the product of the achievable rate on PRB $n$ if it is assigned to UE $m\left(r_{m, n}\right)$ and the current demand factor of UE $m\left(d_{m}\right)$, when both intra-eNB interferers are active. The demand factor of a UE is defined as the ratio of the received throughput to average sector throughput. When an intra-eNB sector is restricted, a penalty (in terms of rate) is introduced in the calculation of the utility as below.

$$
u_{n, m}=\left\{\begin{array}{c}
r_{n, m} \times d_{m} ; \text { all transmit } \\
\left(r_{n, m}-r_{p}\right) \times d_{m} ; \text { one restricted }
\end{array}\right.
$$

where $r_{p}$ is the rate penalty considered in the utility measure to account for resource loss due to restriction imposed to one of the intra-eNB sectors. In simulations, $r_{p}=1.5 \mathrm{bps} / \mathrm{Hz}$ is considered such that average number PRBs restricted by intraeNB avoidance algorithm is around $10 \%$ of the system PRBs. It should be noted that in calculating $r_{m, n}$, all other inter-eNB interferers are considered active.

Entries in $U_{\text {intra }}$ corresponding to UEs having demand factors less than 1 (i.e., rate satisfied in the past) are set to zero so that restrictions are made only for the rate deprived UEs. Then, the Hungarian algorithm is applied to $U_{\text {intra }}$. In each iteration, algorithm selects best PRBs for UEs in three different sectors so that the sum of utilities of the chosen PRBs is maximized. Note that an entry from a matrix with restriction is chosen only when the rate improvement due to interference suppression exceeds the penalty $r_{p} . U_{\text {intra }}$ is updated after each iteration as follows.

- If a chosen entry is from the matrix where PRB restriction is not required, for example $U_{2,3}^{1}$, utility entries for the corresponding PRB for all UEs in $U_{2}^{1}, U_{1}^{2}, U_{3}^{2}, U_{2}^{3}, U_{3}^{1}$, and $U_{1}^{3}$ in addition to $U_{2,3}^{1}$ would have to be replaced by zeros. However, utility entries in $U_{1,3}^{2}$ and $U_{1,2}^{3}$ remain unchanged in order to allow future iteration to reselect this PRB.

- If a chosen entry is from the matrix where a PRB restriction is required, for example $U_{3}^{1}$ (sector 2 has restriction on the selected $\mathrm{PRB}$ ), following actions are required.

o Place the PRB index in restriction list for sector 2

o Entries corresponding to this PRB for all UEs in all matrices except $U_{1}^{3}$ have to be replaced by zeros. In this case, future iterations will allow this PRB to be used only by a UE in sector 3 .

The above steps are repeated until all entries in $U_{\text {intra }}$ are zero. In this process, each sector prepares PRB restrictions for its intra-eNB neighbours. The number of required iterations varies depending on the number of rate deprived UEs as well the utility values of the matrix.

\section{$B$. Inter-eNB inter-cell interference avoidance}

Similar to [10], each sector prepares PRB restrictions for inter-eNB interferers, based on dominant received interference from four inter-eNB interferers. This algorithm involves preparation of utility matrix $U_{\text {inter }}$ by using heuristics and applying Hungarian assignment algorithm to this matrix. Then, neighbouring eNBs are communicated about these resource restrictions over $\mathrm{X} 2$ interface. The details of this algorithm are discussed below.

\section{1) Preparation of utility matrix}

Let us consider $r_{m, n_{(\min )}}^{(i)}$ and $r_{m, n_{\text {(max }}}^{(i)}$ to be the achievable rates for UE $m$ and PRB $n$ at sector $i$ when none and all 1st-tier inter-eNB interferers are restricted, respectively. However, 
moving from $r_{m, n_{(\min )}}^{(i)}$ to $r_{m, n_{(\max )}}^{(i)}$ implies increasing penalty to the interfering sectors, as more and more interferers are to be restricted.

A threshold-based strategy is used to determine which interferers are to be restricted. Based on its demand factor and channel conditions, a UE can restrict two most dominant interferers at most. This limits the number of resulting PRB restrictions in the neighbouring sectors. In order to construct inter-eNB utility matrix, the following steps are repeated for each UE and PRB.

- Four inter-eNB dominant interferers are sorted in descending order into a dominant interferer set.

- $r_{m, n_{(\min )}}^{(i)}$ is calculated considering the presence of all intercell inter-eNB dominant interferers and taking intra-eNB restrictions into account.

○ If $r_{m, n_{\text {(min) }}}^{(i)} \geq r_{1}^{T H}$, no interferers are to be restricted if PRB $n$ is assigned to UE $m$ irrespective of its demand factor. In this case, UE $m$ is then either having a strong desired link from serving BS or is experiencing weak interference from all dominant interferers on PRB $n$. In simulations, we have used $r_{1}^{T H}=3.5 \mathrm{bps} / \mathrm{Hz}$.

○ Else, calculate the new rate $r_{m, n_{(\text {new }}}^{(i)}$ with the most dominant interferer being restricted.

- If $r_{m, n_{\text {(new })}}^{(i)}-r_{m, n_{\text {(min) }}}^{(i)} \geq r_{2}^{T H}$, UE $m$ will request this dominant interferer to be restricted irrespective of its demand. $r_{2}^{T H}=2 \mathrm{bps} / \mathrm{Hz}$ has been used in simulations.

- Else if $d_{m}^{(i)}>1$ (UE $m$ has been rate derived in the past), $r_{m, n_{\text {(min) }}}^{(i)}=0$, and $r_{m, n_{(n e w)}}^{(i)}>0$, the most dominant interferer is to be restricted. In some cases, both $r_{m, n_{\text {(min) }}}^{(i)}$ and $r_{m, n_{\text {(new })}}^{(i)}$ can be zero. If restricting two most dominant interferers provides achievable rate, these will be marked for restriction.

Note that the above threshold values are chosen such that the number of PRBs restricted by inter-eNB algorithm is on average around $15-20 \%$ of the available system bandwidth. After finding the inter-eNB dominant interferer(s) to be restricted on each PRB and each UE, achievable rates $r_{m, n}^{(i)}$ are calculated. Now, the utility of PRB $n$ for UE $m$ can be expressed as:

$$
u_{m, n}^{(i)}=r_{m, n}^{(i)} \times d_{m}^{(i)} .
$$

The utility matrix is given by $U_{\text {inter }}^{(i)}=\left[u_{m, n}^{(i)}\right]$. Each entry of $U_{\text {inter }}^{(i)}$ is associated with corresponding interferer(s) to be restricted in addition to the achievable rate and demand when $\operatorname{PRB} n$ is used by UE $m$.

\section{2) Applying Hungarian algorithm to utility matrix}

Hungarian algorithm is applied to $U_{\text {inter }}^{(i)}$ in an iterative manner similar to Section III(A). In each sector, steps given below are followed to prepare inter-eNB PRBs restrictions.

- Apply Hungarian algorithm to $U_{\text {inter }}^{(i)}$. If any selected utility entry has a corresponding interferer restriction, the restriction list will be updated with the marked interferer for the PRB.

- Update the columns of the utility matrix corresponding to assigned PRBs with zeros. Now, apply the Hungarian algorithm to the updated utility matrix.

- Repeat above steps until all entries of the utility matrix are zero. The number of required iterations is bounded by $\lceil N / M\rceil$.

\section{3) Inter-eNB communication using X2 interface}

For a particular PRB, Fig. 3 shows an example scenario of inter-eNB inter-cell restrictions. In this figure, the green (solid line) and red (dashed line) arrows indicate that inter-eNB inter-cell interference received at the arrow-originating-sector from the arrowhead-sector is acceptable and unacceptable (to be restricted), respectively. For example, for a PRB of interest, sector $B$ can tolerate interference from sector $A$, but the opposite is not true as there is a red (dashed) arrow from sector $A$ toward $B$. In this case, either sector $A$ or $B$ has to be restricted for this PRB. In this case, eNB corresponding to sector $A$ communicates with the eNB corresponding to sector $B$ using X2 interface about restricting the PRB.

It is expected that a pair of sectors will have restrictions for the same PRB in some cases (i.e., red arrows to each other). In such cases, the sector that achieves higher utility survives. The negotiation of resolution of this type of conflicting restrictions is also carried over X2 interface.

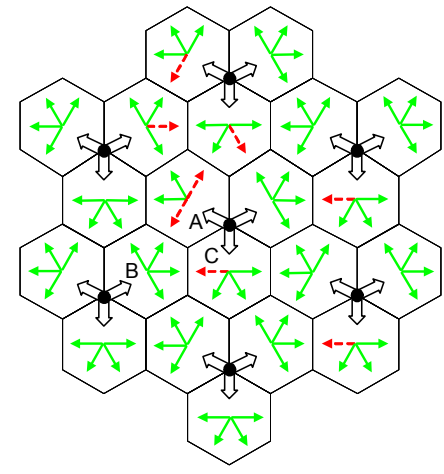

Fig. 3: Graphical representation of inter-eNB PRB restriction

\section{SimUlation SYSTEM AND PARAMETERS}

Considered simulation and system parameters are taken mostly from [1] and [17] as summarized in Table I.

Time-frequency correlated 6-taps extended spatial channel model (SCME) with power delay profile as defined in [18] is considered. Independent lognormal shadow fading with a standard deviation of $8 \mathrm{~dB}$ has been assumed. 
$20 \mathrm{MHz}$ system bandwidth constitutes 100 PRBs. A PRB consists of 12 subcarriers (each of $15 \mathrm{kHz}$ ) in frequency and 7 OFDM symbols in the time dimension. We assume that 4 and 3 OFDM symbols per PRB are used for downlink reference and control signals, respectively, giving 77 OFDM symbols per PRB for data traffic. Therefore, a PRB can carry 77 information bits with QPSK rate 1/2 modulation and coding scheme (MCS).

TABLE I

SIMULATION PARAMETERS

\begin{tabular}{|c|c|}
\hline Parameter & Assumption \\
\hline Cellular layout & $\begin{array}{c}\text { Hexagonal grid, } 19 \text { cell sites, } 3 \text { sectors } \\
\text { per site }\end{array}$ \\
\hline Inter-site distance & $500 \mathrm{~m}$ \\
\hline Carrier frequency / Bandwidth & $2.0 \mathrm{GHz} / 20 \mathrm{MHz}$ (100 PRBs) \\
\hline Distance-dependent path loss & $L=128.1+37.6 \log _{10}(R)$ \\
\hline Lognormal shadowing & Independent among links \\
\hline Shadowing standard deviation & $8 \mathrm{~dB}$ \\
\hline UE speeds of interest & $30 \mathrm{~km} / \mathrm{hr}$ \\
\hline Penetration loss & $10 \mathrm{~dB}$ \\
\hline Antenna pattern (horizontal) & $\begin{array}{l}A(\theta)=-\min \left[12\left(\frac{\theta}{\theta_{3 d B}}\right)^{2}, A_{m}\right] \\
\theta_{3 d B}=70 \text { degrees, } A_{m}=20 \mathrm{~dB}\end{array}$ \\
\hline Antenna configuration & Single-Input-Single-Output \\
\hline BS antenna gain & $14 \mathrm{dBi}$ \\
\hline UE antenna gain & $0 \mathrm{dBi}$ \\
\hline UE noise figure & $7 \mathrm{~dB}$ \\
\hline AMC modes & $\begin{array}{l}\text { QPSK, 16-QAM, and 64-QAM with } \\
\text { varying rates }\end{array}$ \\
\hline Channel model & 6-Tap SCME \\
\hline Total sector TX power & $46 \mathrm{dBm}$ \\
\hline $\begin{array}{c}\text { Downlink inter-cell interference } \\
\text { modelling }\end{array}$ & $\begin{array}{l}\text { Explicit modelling (all links are } \\
\text { simulated) }\end{array}$ \\
\hline $\begin{array}{c}\text { Minimum distance between UE } \\
\text { and BS }\end{array}$ & $35 \mathrm{~m}$ \\
\hline Traffic model & Full buffer \\
\hline Scheduler & $\begin{array}{c}\text { Hungarian algorithm based on utility } \\
\text { matrix }\end{array}$ \\
\hline
\end{tabular}

Adaptive modulation and coding (AMC) is used with various MCS modes with quadrature phase shift keying (QPSK), 16-, and 64-quadrature amplitude modulation (QAM), and coding rates ranging from $1 / 8$ to $4 / 5$. Link adaptation is performed using attenuated and truncated form of Shannon bound, matched to link level performance curves with above mentioned modulation level and coding rates, as follows [19].

$$
\eta=\left\{\begin{array}{cc}
0 & ; \gamma<\gamma_{\min }, \\
\alpha \mathrm{S}(\gamma) & ; \gamma_{\min }<\gamma<\gamma_{\max }, \\
\eta_{\max } & ; \gamma>\gamma_{\max },
\end{array}\right.
$$

where $\eta$ is the spectral efficiency in $\mathrm{bps} / \mathrm{Hz}, \gamma$ is the signal-tointerference-plus-noise ratio (SINR) seen on PRB and $\alpha(0.75$ used in simulation) is the attenuation factor applied to the Shannon bound given by $S(\gamma)=\log _{2}(1+\gamma)$ which achieves $\eta_{\max }(4.8 \mathrm{bps} / \mathrm{Hz})$ at $\gamma_{\max }(19.2 \mathrm{~dB})$ or beyond and 0 at $\gamma_{\min }(-$ $6.5 \mathrm{~dB}$ ) or lower. Automatic repeat request (ARQ) has not been considered in simulations.
For fair comparison, total transmit power per sector is kept constant in all schemes, which is $\approx P_{t}$. Accordingly, power allocated to PRBs for different schemes are shown in Table II. In the table, $P_{t}(46 \mathrm{dBm}), N(100), N_{\text {res }}$, and $N_{\text {elig }}$ are the total power per sector, system bandwidth in terms of number of PRBs, and the number of restricted (from intra and inter-eNB), and unrestricted PRBs, respectively. As shown in the table, restricted PRBs are unused in proposed scheme 1 and used with $10 \mathrm{~dB}$ lower power in proposed scheme 2 .

TABLE II

POWER ALLOCATION FOR DIFFERENT SCHEMES

\begin{tabular}{c|c}
\hline Scheme & Allocated power on PRBs \\
\hline \hline Reuse 1 & $P_{r b}^{R 1}=P_{t} / N$ \\
\hline Reuse 3 & $P_{r b}^{R 3}=3 P_{t} / N$ \\
\hline PFR eff reuse & $P_{r b, \text { out }}^{P F R 1.3}=2.25 P_{t} / N, P_{r b, \text { in }}^{P F R 1.3}=1.13 P_{t} / N$ \\
\hline 1.3 & $P_{r b, \text { out }}^{S F R 1.5}=1.5 P_{t} / N, P_{r b, \text { in }}^{S F R 1.5}=0.75 P_{t} / N$ \\
\hline $\begin{array}{c}\text { SFR eff reuse } \\
1.5\end{array}$ & $P_{r b, \text { out }}^{S F R 2.0}=2.0 P_{t} / N, P_{r b, \text { in }}^{S F R 2.0}=0.5 P_{t} / N$ \\
\hline $\begin{array}{c}\text { SFR eff reuse } \\
2.0\end{array}$ & $P_{r b, \text { out }}^{S F R 2.5}=2.5 P_{t} / N, P_{r b, \text { in }}^{S F R 2.5}=0.25 P_{t} / N$ \\
\hline SFR eff reuse & $P_{r b, \text { out }}^{S F R 2.75}=2.75 P_{t} / N, P_{r b, \text { in }}^{S F R 2.75}=0.13 P_{t} / N$ \\
\hline $\begin{array}{c}\text { SFR eff reuse } \\
2.75\end{array}$ & $P_{r b, \text { elig }}^{P R O P 1}=P_{t} /\left(N-N_{r e s}\right), P_{r b, r e s}^{P R O P 1}=0$ \\
\hline $\begin{array}{c}\text { Proposed } \\
\text { scheme 1 }\end{array}$ & $P_{r b, \text { elig }}^{P R O P 2}=10 P_{t} /\left(10 N_{\text {elig }}+N_{r e s}\right), P_{r b, \text { res }}^{\text {PROP2 }}=P_{t} /\left(10 N_{\text {elig }}+N_{r e s}\right)$ \\
\hline $\begin{array}{c}\text { Proposed } \\
\text { scheme 2 }\end{array}$
\end{tabular}

\section{Simulation Results AND Discussions}

The performance of the proposed interference avoidance scheme is compared to that of the reference schemes in terms of cell-edge and average sector throughput. Cell-edge throughput is defined as the $5^{\text {th }}$ percentile point of CDF of UE throughput. The reference schemes simulated are reuse 1, reuse 3, SFR (with effective reuse of 1.5, 2.0, 2.5, and 2.75), and PFR (effective reuse of 1.3).

Statistics are collected from a total of 300 drops. In each drop, UEs are uniformly distributed according to a density of $12 \mathrm{UEs} / \mathrm{sector}$. Simulation time span is $25 \mathrm{~ms}$ (50 OFDM symbols) in each drop. Resource restriction refreshment interval considered is 6 OFDM symbols (within the channel coherence time for the UE speed of $30 \mathrm{~km} / \mathrm{hr}$ ).

UEs are placed in the central 21 sectors among the total 57 sectors of 19 sites. For the proposed scheme, allocation algorithms are run in these 21 sectors. Statistics are collected from the central eNB (3 sectors) only. Interference is calculated using central cell approach.

Fig. 4 shows CDF of UE throughput for the proposed as well as reference schemes. The lower tail of CDF is zoomed in Fig. 5 in order to view the cell-edge throughput clearly. Fig. 6 compares cell-edge and average sector throughput among all simulated schemes with reference to reuse 1 scheme. It is observed that while reuse 3 scheme achieves $168.4 \%$ improvement in cell-edge throughput compared to reuse 1 scheme, it suffers from sector throughput degradation by $49.2 \%$. The PFR scheme improves cell-edge throughput by $149.7 \%$, however, with $26 \%$ reduction in sector throughput. 
SFR schemes with effective reuse of 2.0, 2.5, and 2.75 show cell-edge throughput improvement by $21.4 \%, 69.4 \%$, and $114.7 \%$ with degradation of sector throughput by $4.3 \%, 8.6 \%$, and $26.1 \%$, respectively. SFR scheme with effective reuse of 1.5 neither improves cell-edge nor sector throughput compared to reuse 1 scheme. Comparing with reuse 1 scheme, proposed scheme 1 improves cell-edge throughput by $266.1 \%$ with an improvement in sector throughput by $3.9 \%$. Proposed scheme 2 shows inferior cell-edge and superior sector throughput compared to those in proposed scheme 1 as restricted PRBs are used with reduced power, which favors cell-centre UEs while harms those at the cell-edge. However, compared to reuse 1 scheme, proposed scheme 2 obtains $171.3 \%$ improvement in cell-edge throughput while improving the sector throughput by $7.2 \%$.

The performance gain in the proposed scheme is achieved with the cost of increased overhead required for UE-BS feedback as well as eNB-eNB communication over X2 interface.

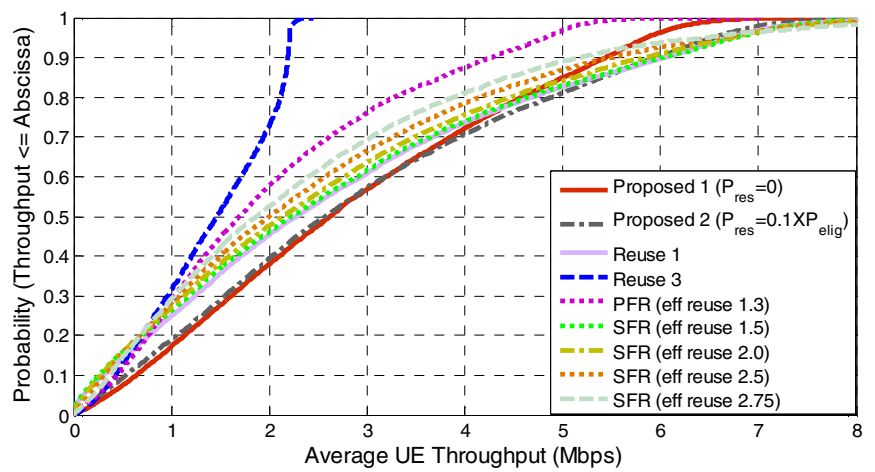

Fig. 4: CDF of UE throughput

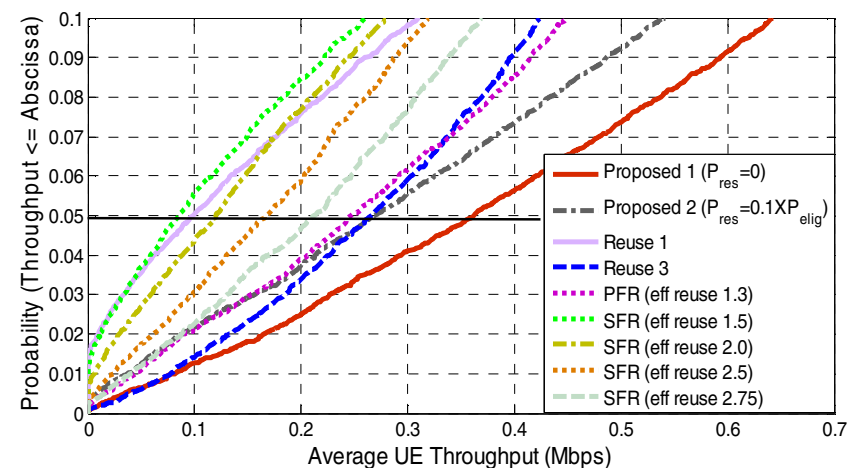

Fig. 5: CDF of UE throughput (zoomed to show cell-edge throughput)

\section{CONCLUSIONS}

We have presented and evaluated an interference avoidance scheme for downlink LTE system that uses dynamic inter-cell coordination supported by X2 interface. Two variations of the proposed scheme have been compared with the reference reuse 1 scheme as well as the static partition based interference avoidance schemes in the literature. It has been observed that although static schemes achieve improved cell-edge throughput, they suffer seriously in terms of sector throughput. On the other hand, the proposed schemes not only achieve higher cell-edge throughput but also show improvement in average sector throughput compared to those in any static scheme.

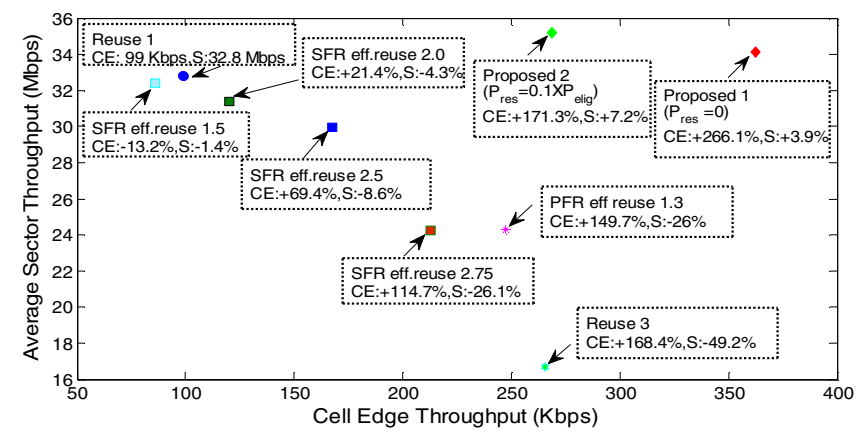

Fig. 6: Cell-edge vs. average sector throughput

\section{ACKNOWLEDGMENT}

This research is financially supported in part by the Communications Research Centre of Canada (CRC).

\section{REFERENCES}

[1] 3GPP TR 25.814 V7.1.0, "Physical Layer Aspects for Evolved Universal Terrestrial Radio Access (UTRA) (Release 7)," September 2006.

[2] WiMAX Technical Report, "Mobile WiMAX - Part I: A Technical Overview and Performance Evaluation," August 2006.

[3] A. Alexiou, "WINNER: Designing a new radio interface for nextgeneration systems," Bell Labs Technical Journal, vol. 2, pp. 19-35, August 2007.

[4] J. Chuang and N. Sollenberger, "Beyond 3G: wideband wireless data access based on OFDM and dynamic packet assignment," IEEE Commun. Mag., vol. 38, pp. 78-87, July 2000.

[5] V.H. MacDonald, "The cellular concept," Bell System Technical Journal, vol. 58, no.1, pp. 15-41, January 1979.

[6] K. Begain, G.I. Rozsa, A. Pfening, and M. Telek, "Performance analysis of GSM networks with intelligent underlay-overlay," in Proc. Seventh International Symposium on Computers and Communications (ISCC 2002), July 2002, pp. 135-141.

[7] 3GPP R1-050738, "Interference Mitigation - Considerations and Results on Frequency Reuse," Siemens, September 2005.

[8] 3GPP R1-060291, "OFDMA Downlink Inter-Cell Interference Mitigation," Nokia, February 2006.

[9] WINNER II Deliverable D4.7.2, "Interference Avoidance Concepts," June 2007, available at http://www.ist-winner.org.

[10] M. Rahman and H. Yanikomeroglu, "Interference avoidance through dynamic downlink OFDMA subchannel allocation using intercell coordination," in Proc. IEEE VTC Spring 2008, pp. 1630-1635, May 2008.

[11] 3GPP TS 36.300 V8.4.0, "E-UTRA and E-UTRAN Overall description; Stage 2 (Release 8),' March 2008

[12] 3GPP R1-050507, "Soft Frequency Reuse Scheme for UTRAN LTE," Huawei, May 2005.

[13] 3GPP R1-050841, "Further Analysis of Soft Frequency Reuse Scheme," Huawei, September 2005.

[14] M. Sternad, T. Ottosson, A. Ahlen, and A. Svensson, "Attaining both coverage and high spectral efficiency with adaptive OFDM downlinks," in Proc. IEEE VTC 2003 Fall, Oct. 2003, pp. 2486-2490.

[15] 3GPP R1-060135, "Interference Mitigation by Partial Frequency Reuse," Siemens, January 2006.

[16] H.W. Khun, "The Hungarian method for the assignment problem," Naval Research Logistic Quarterly, vol. 2, pp. 83-97, 1955.

[17] 3GPP TS 36.211 V8.2.0, "EUTRA Physical Channels and Modulation (Release 8)," March 2008.

[18] D.S. Baum, J. Salo, M. Milojevic, P. Kyösti, and J. Hansen, "An interim channel model for beyond-3G systems," in Proc. IEEE VTC 2005 Spring, pp. 3132 - 3136, May 2005.

[19] 3GPP TR 36.942 V1.2.0, "E-UTRA Radio Frequency (RF) System Scenarios (Release 8), June 2007. 